\title{
Mortalidade por sifilis nas regiões brasileiras, 1980-1995
}

\author{
Mortality by syphilis in the Brazilian regions, 1980-1995
}

Bruno Gil de Carvalho Lima

\section{unitermos resumo}

Sifilis congênita

Mortalidade

VDRL
Introdução: A sífilis tem diminuído sua prevalência após o advento da antibioticoterapia.

No Brasil, é objetivo do Ministério da Saúde a erradicação, daí recomendar-se seu rastreamento mediante testes não-treponêmicos e o tratamento mesmo na impossibilidade de confirmar o diagnóstico. O pré-natal é um momento importante, principalmente para o combate às formas congênitas. Objetivo: Estudar a evolução da mortalidade por sífilis congênita e não-congênita nas regiões brasileiras de 1980 a 1995. Metodologia: O número de óbitos foi conseguido a partir do Datasus. Foram calculados coeficientes de mortalidade por lues para cada macrorregião brasileira. Resultados: Houve uma tendência comum de queda dos coeficientes de mortalidade de 1980 a 1995, embora as regiões Nordeste e Norte tenham apresentado taxas ascendentes no primeiro qüinqüênio. A mortalidade pelas formas não-congênitas, estando em declínio, oscilou entre valores muito baixos. Sífilis congênita apresentou coeficientes mais elevados, entre 1,33 e 8,87/100.000. A região Nordeste apresentou elevação do coeficiente de $120 \%$ de 1980 a 1990, com queda discreta (11,5\%) até 1995. Discussão: Os esforços no sentido de diminuir a prevalência de sífilis no Brasil parecem ter resultado em avanços, já que os coeficientes de mortalidade tenderam ao decréscimo. Entretanto, as baixas taxas de mortalidade são um indicador não apenas da freqüência de sífilis, mas da sua letalidade, bem menor atualmente. Conclusões: Chama a atenção a persistência de níveis elevados, até ascendentes, de mortalidade por lues congênita, provavelmente pelas deficiências existentes na assistência perinatal, que ocorre de forma desigual no território nacional.

\section{abstract}

Background: The prevalence of syphilis has decreased since the discovery of antibiotics. In Brazil, the Ministry of Health intends to eradicate the disease, thus recommending the screening with non-treponemic tests and treatment even when it is not possible to confirm the diagnosis.

Antenatal care constitutes an important chance to do so, targeting the prevention of congenital forms. Objective: Study the evolution of mortality by congenital and non-congenital syphilis in the Brazilian regions from 1980 to 1995. Methods: The number of deaths was taken from Datasus. Mortality rates by syphilis in each Brazilian region were calculated. Results: There was a common decreasing trend of the rates from 1980 to 1995, even though the North and Northeast regions showed increasing rates in the first five years. The mortality by non-congenital forms declined, oscillating between very low values. Congenital syphilis had more elevated rates, between 1,33 and 8,87/100.000. The Northeastern region presented an increase of 120\% from 1980 to 1990, with a little fall $(11,5 \%)$ until 1995. Discussion: The efforts to diminish the prevalence of syphilis in Brazil seem to have succeeded to some extent, since the mortality rates tended to decrease. Even though, not only are the low rates indicative of its frequency, they also reflect its lethality, which is much lower nowadays. Conclusions: The persistence of elevated, and even increasing, levels of mortality by congenital syphilis is probably a consequence of the deficiencies of antenatal care, which are unevenly distributed throughout the national territory.

\section{key words}

Congenital syphilis

Mortality

VDRL
Faculdade de Medicina, Universidade Federal da Bahia. Trabalho apresentado no XII Congresso Latino-Americano de Doenças Sexualmente Transmisśiveis e VI Conferência Pan-Americana de Aids com 0 título Evolução da Mortalidade por Sifilis nas Regiões Brasileiras, 1980-1995: o Problema da Sífilis Congênita, em Salvador, 1999. 


\section{Introdução}

A sífilis já ocupou um lugar de grande importância entre as enfermidades sexualmente transmissíveis, quando era comum encontrarem-se casos avançados (formas secundária e terciária), responsáveis por um sem-número de óbitos, fato piorado pela impossibilidade de tratá-la. O advento da antibioticoterapia mudou muito a situação da doença, possibilitando a cura e a interrupção da sua evolução, passando a haver um predomínio das suas formas precoces e uma diminuição considerável da sua mortalidade.

Com este avanço, aliado ao fato de que não há registro de resistência do Treponema pallidum à penicilina, criouse um ambiente de otimismo que redundou no compromisso dos países americanos de erradicar a doença do continente até o ano 2000. Entretanto nenhuma das nações envolvidas conseguiu fazê-lo, por motivos diversos, segundo a realidade de saúde de cada uma.

Trabalhos disponíveis acusam uma prevalência em torno de $5 \%$ na população geral $(2,6,10)$, com níveis compreensivelmente mais elevados em grupos de risco aumentado, como internos de instituições penitenciárias (7) e prostitutas (12), e menores em subpopulações mais jovens e com melhor estado de higidez, como os convocados para o serviço militar (15).

No Brasil, costumam-se utilizar métodos diagnósticos não-treponêmicos, como o venereal diseases research laboratory (VDRL), mais usado, e o rapid plasma reagin (RPR), para rastreamento, solicitando-se testes de maior especificidade, como o FTA-Abs, quando há positividade da reação sorológica inicial. Os testes de rastreamento são baratos, possíveis de se realizar com estrutura laboratorial simples e apresentam alta sensibilidade, favorecendo o diagnóstico precoce e o tratamento em tempo hábil para evitar as seqüelas das formas tardias da sífilis. Por outro lado, tem havido esforços no sentido de favorecer a utilização de métodos de maior reprodutividade, como a hemaglutinação passiva (14), em substituição à FTA-Abs, cuja realização ainda não é prontamente conseguida em certas regiões do país.

A assistência pré-natal constitui um momento de especial importância para medidas visando a diminuir a prevalência da sífilis (9), por se tratar de um período em que muitas mulheres que permanecem à margem do sistema de saúde procuram instituições a fim de garantir um desfecho sem complicações para a gestação. Mais ainda, é nesta oportunidade que se pode evitar o problema da sífilis congênita, forma da doença associada a grande risco gestacional $(4,11)$, com quadro que já se instala de forma equivalente à da lues tardia desde o início (13). Quando não termina em óbito fetal, a sífilis congênita provoca seqüelas graves para o recém-nascido. Trabalho anterior apontou tratamento inadequado como causa freqüente de sífilis congênita (8), mas a falha em solicitar uma sorologia no $2^{\circ}$ e $3^{\underline{0}}$ trimestres após um resultado não-reagente no início do pré-natal também colabora para a manutenção de níveis inaceitáveis de incidência e mortalidade por tal enfermidade no Brasil.

Como parte do compromisso assumido de erradicar a doença, preconiza-se no Brasil o rastreamento para todas as gestantes com os testes não-treponêmicos e o tratamento mesmo quando não é possível confirmar o diagnóstico, mas tais medidas não têm conseguido combater adequadamente o problema, provavelmente por falta de real implementação das orientações do Ministério da Saúde na ponta do sistema (5).

\section{Objetivos}

Calcular os coeficientes de mortalidade por sífilis, suas formas congênitas e não-congênitas, nas regiões brasileiras nos anos de 1980, 1985, 1990 e 1995 e analisar a tendência temporal das taxas nos quinze anos estudados, por região.

\section{Material e método}

Dados referentes ao número de óbitos por sífilis foram conseguidos a partir do Sistema de Informações de Mortalidade do Sistema Único de Saúde, Ministério da Saúde (SIMDatasus), utilizando-se o programa TABX. Selecionaram-se os óbitos pelas causas 090 a 097, segundo a Classificação Internacional de Doenças - $9^{\underline{a}}$ Revisão, agrupados de acordo com as cinco macrorregiões, analisando-se informações sobre sexo e idade. Foram incluídos no estudo apenas os óbitos ocorridos em 1980, 1985, 1990 e 1995.

A população em cada ano estudado foi retirada dos censos de 1980, 1991 e contagem populacional de 1996 estimando-se as populações nos anos intercensitários pelo método da interpolação geométrica, informações coletadas na Fundação Instituto Brasileiro de Geografia e Estatística. Foram calculados coeficientes de mortalidade por milhão de habitantes devido a lues (formas não-congênitas) para cada uma das macrorregiões brasileiras nos anos em estudo. 
Embora fosse mais adequado usar como denominador dos coeficientes de mortalidade por sífilis congênita o número de nascidos vivos, persistem no Brasil dificuldades quanto ao registro de nascimentos, sendo esta informação indisponível em diversos estados. Optou-se por utilizar como base dos coeficientes a população de menores de um ano, que representa uma aproximação aceitável do número de nascidos vivos, sendo comumente utilizada em estudos de mortalidade infantil.

Compararam-se os resultados quanto à sua distribuição nas regiões brasileiras para evitar o problema da oscilação de coeficientes, que poderia ocorrer ao se estudar estados pouco populosos, e diminuir o número de unidades de comparação, que seria muito grande ao se tomar cada estado separadamente. Foi dada maior importância à evolução temporal dos coeficientes no período de 1980 a 1995.

Não foi necessário o uso de testes de significância estatística ou outros métodos matemáticos por se tratar de estudo descritivo, sem o objetivo de testar, mas, sim, de levantar hipóteses.

\section{Resultados}

O coeficiente de mortalidade por sífilis no Brasil foi de 2,45/1.000.000 habitantes em 1980, diminuindo continuamente até níveis de 1,02/1.000.000 em 1995, um decréscimo de $58,2 \%$ em 15 anos. Houve uma tendência comum a todas as regiões de queda dos coeficientes de mortalidade por sífilis de 1980 a 1995, embora as regiões Nordeste e Norte tenham apresentado taxas ascendentes no primeiro qüinqüênio (Tabela 1).

A mortalidade pelas formas não-congênitas de lues, além de estar em declínio, oscilou entre 0,8/1.000.000 (região NE, 1980) e 0,04/1.000.000 (região Sul, 1995), conforme Tabela 2. Mais de 67\% foram de pessoas do sexo masculino.

Coeficientes de mortalidade por sifilis nas regiöes brasileiras por

Tabela 1 milhão de habitantes, 1980 a 1995

\begin{tabular}{lllll}
\hline \multicolumn{1}{r}{ Ano } & 1980 & 1985 & 1990 & 1995 \\
Região & & & & \\
Norte & 1,02 & 3,22 & 0,83 & 1,27 \\
Nordeste & 2,01 & 2,34 & 2,09 & 1,65 \\
Sudeste & 2,90 & 2,24 & 1,28 & 0,79 \\
Sul & 2,68 & 1,72 & 1,19 & 0,60 \\
Centro-0este & 1,86 & 1,04 & 0,87 & 0,49 \\
\hline
\end{tabular}

A sífilis congênita apresentou coeficientes mais elevados, o máximo de 8,87/100.000 menores de 1 ano na região Norte em 1985, e o mínimo de 1,33/100.000 menores de 1 ano na mesma região em 1980. A região Nordeste apresentou elevação do coeficiente de $120 \%$ de 1980 a 1990, com queda discreta (11,5\%) até 1995 (Tabela 3). Os óbitos se concentraram no primeiro ano de vida (98\%), sendo que $30,8 \%$ ocorreram ainda no primeiro dia após o nascimento e, ao final do primeiro mês de vida, apenas $18,8 \%$ das crianças sobreviveram.

\section{Discussão}

\section{Evolução da mortalidade por sífilis}

O quadro descrito pela tendência temporal descendente dos coeficientes de mortalidade por sífilis é muito auspicioso no país como um todo. Um decréscimo de quase $60 \%$ num espaço de 15 anos é um avanço muito grande, principalmente quando se atenta para o fato de que nenhum método diagnóstico ou terapêutico novo

Coeficientes de mortalidade por sifilis (exceto formas congênitas) nas regiöes brasileiras por milhão

Tabela 2 de habitantes, 1980 a 1995

\begin{tabular}{|c|c|c|c|c|}
\hline Ano & 1980 & 1985 & 1990 & 1995 \\
\hline \multicolumn{5}{|l|}{ Região } \\
\hline Norte & 0,51 & 0,28 & 0,21 & 0,36 \\
\hline Nordeste & 0,80 & 0,51 & 0,14 & 0,13 \\
\hline Sudeste & 0,58 & 0,39 & 0,34 & 0,15 \\
\hline Sul & 0,47 & 0,44 & 0,14 & 0,04 \\
\hline Centro-0este & 0,26 & 0,35 & 0,55 & 0,10 \\
\hline
\end{tabular}

Tabela 3 de 1 ano, 1980 a 1995

\begin{tabular}{|c|c|c|c|c|}
\hline Ano & 1980 & 1985 & 1990 & 1995 \\
\hline \multicolumn{5}{|l|}{ Região } \\
\hline Norte & 1,33 & 8,87 & 2,17 & 3,42 \\
\hline Nordeste & 3,53 & 6,20 & 7,78 & 6,88 \\
\hline Sudeste & 8,74 & 8,02 & 4,73 & 3,62 \\
\hline Sul & 8,38 & 5,35 & 4,88 & 2,84 \\
\hline Centro-0este & 5,06 & 2,51 & 3,24 & 1,82 \\
\hline
\end{tabular}


passou a contribuir para um combate mais efetivo da enfermidade, restando como explicação uma melhor qualidade de rastreamento e tratamento da sífilis. Ressaltese que as duas regiões menos favorecidas do país (Norte e Nordeste) foram as que mais demoraram a participar dessa tendência à diminuição da mortalidade, chegando a apresentar tendência ao incremento de 1980 para 1985. No período estudado, as regiões Sul, Sudeste e CentroOeste apresentaram melhoria da mortalidade de mais de $70 \%$, chegando a $77 \%$ na região Sul, que, embora não disponha dos mesmos recursos dos estados do Sudeste, tem vários outros indicadores positivos de saúde e boa organização da rede de serviços públicos. O Norte terminou os 15 anos em análise com descenso pequeno, de $17 \%$. Os estados nordestinos, ao contrário, demonstraram aumento do coeficiente de 1990 a 1995. Não se pode dizer, com base no presente estudo, se tal tendência foi sustentada nos anos seguintes ou se foi achado isolado, mas este fato é tema interessante para investigações futuras.

\section{Sífilis não-congênita}

O comportamento da mortalidade pelas formas nãocongênitas de sífilis foi compatível com o que se depreende nos atendimentos cotidianos em ambulatórios e serviços especializados. A possibilidade de diagnosticar e tratar a doença de modo relativamente fácil e barato tem levado a uma diminuição considerável da mortalidade, a qual muito contribuiu para a situação descrita para mortalidade por sífilis como um todo. Os baixos coeficientes podem ser comemorados como êxito parcial na luta contra a sífilis, principalmente ao se perceber que os avanços se distribuíram por todas as regiões do país.

\section{Sífilis congênita}

O problema da sífilis congênita constituiu o lado mais preocupante da patologia. Nas regiões onde houve decréscimo da mortalidade, não foi tão pronunciado como para as formas adultas da doença, além de terem-se mantido coeficientes mais elevados ao fim dos anos analisados. As regiões Norte e Centro-Oeste não conseguiram demonstrar uma tendência declinante estável, cursando com oscilações consideráveis a cada qüinqüênio. O Nordeste apresentou a pior situação, com aumento muito grande, de 120\%, no decênio de 1980 a 1990, caindo apenas $11,5 \%$ nos cinco anos seguintes e mantendo, ao fim do período estudado, o mais alto coeficiente de mortalidade por sífilis congênita do Brasil.

\section{Prevalência $x$ letalidade}

Os resultados apontam uma situação muito preocupante: como não há outra maneira de transmissão do Treponema pallidum de modo a causar a forma congênita da lues que não a vertical, cada caso corresponderá a uma mãe infectada, provavelmente na fase latente da doença, e possivelmente a um parceiro sexual (ou mais) também portador da espiroqueta. Logo, o quadro de tendência ao controle da sífilis evocado pela mortalidade geral e formas adultas deve ser falacioso. Na verdade, esta é uma limitação do desenho deste estudo.

A mortalidade é resultante da prevalência, mas também da letalidade da doença. Aparentemente, o que tem concorrido para a baixa da mortalidade por sífilis é o segundo fator, que sabidamente diminuiu devido às já mencionadas possibilidade de tratamento e interrupção da evolução natural da patologia. Já a prevalência parece persistir mais elevada, explicando os novos casos de lues que acometem recém-nascidos a cada ano no Brasil. A faceta mais grave do problema não é a manutenção da sífilis como doença sexualmente transmissível prevalente em nosso meio, mas a constatada falha na prevenção da transmissão vertical, que provoca casos muito mais graves da doença e aumenta as taxas de gestação de risco na população.

A rapidez com que se chega ao desfecho fatal após o nascimento, perdendo-se grande parte dos neonatos no primeiro dia e quase todos ao fim do primeiro ano de vida, é um indicador da dificuldade de se tratar a sífilis congênita, reforçando a necessidade de prevenir sua ocorrência. É preciso destacar que não foram computadas as gestações que não foram a termo devido a lues congênita, e que, somadas aos óbitos infantis, resultariam em coeficientes ainda piores.

\section{Disparidades regionais}

$\mathrm{O}$ achado de realidades discrepantes entre as diversas regiões do país é reflexo das diferenças que persistem entre elas nas deficiências do setor de saúde. A estruturação e a disponibilidade, tal como existem em outros países (1), de uma rede de serviços públicos de saúde voltada para o rastreamento e busca ativa de casos (aliados ao pronto tratamento) de várias doenças que muito contribuem para o mau estado de saúde de nossa população levariam a uma redução significativa da morbimortalidade por tais agravos em todo o país, sobretudo nos estados mais pobres, concentrados no Nordeste e no Norte brasileiros. 
É necessário inserir o tema de forma mais sistemática nos currículos das escolas médicas (3) e estimular a educação continuada dos profissionais envolvidos no cuidado pré-natal e assistência obstétrica, de forma a promover amplo rastreamento da sífilis, tratamento adequado e acompanhamento pós-terapêutico das pacientes e seus parceiros, para que se consiga efetivamente alcançar 0 objetivo traçado de eliminar a sífilis congênita do país.

\section{Conclusões}

Verificou-se uma tendência ao declínio da mortalidade por sífilis em todas as regiões brasileiras, sobretudo no tocante às formas adultas da doença.
A mortalidade por sífilis congênita, entretanto, não diminuiu de forma tão intensa, nem foi mantida, chegando a aumentar consideravelmente em estados mais pobres.

É possível que o decréscimo da mortalidade por sífilis seja conseqüência da diminuição da letalidade da doença pela possibilidade de tratamento, mas níveis inaceitavelmente elevados de prevalência vêm se mantendo.

Educação continuada e programas de reciclagem de recursos humanos em saúde constituem medidas importantes para assegurar a correta assistência às gestantes, necessária para a resolução do problema da sífilis congênita.

\section{Referências}

I. Alfei, B. \& Maurel, D.L. Investigación de contactos en enfermos de sífilis en Mar del Plata,Argentina. Bol. Oficina Sanit. Panam., 95(3): 253-60, 1983

2. Andrade, A.L.S.S. et al. Rastreamento sorológico para doenças infecciosas em banco de sangue como indicador de morbidade populacional. Rev. Saúde Pública, 23(I): 20-5, 1989.

3. Baldy, J.L.S. Doenças infecciosas e parasitárias transmitidas por contato sexual: aspectos epidemiológicos e de saúde pública. Rev. Assoc. Méd. Bras., 3 I (9/10): |46-5।, 1985.

4. Duarte, G. et al. Feto morto: aspectos conceituais e etiopatogênicos; análise de 437 casos. Rev. Bras. Ginecol. Obstet., 7(3): 1 15-8, 1985.

5. Duarte, G. et al. Morte fetal por sífilis: avaliação epidemiológica realizada em Ribeirão Preto, Brasil. Bol. Oficina Sanit. Panam., I / 6(4): 290-7, 1994.

6. Ferraroni, J.J. \& Lacaz, C.S. Prevalência de anticorpos contra os agentes causadores da hepatite, malária, sífilis e toxoplasmose em cinco populações humanas distintas da Amazônia brasileira. Rev. Inst. Med. Trop. São Paulo, 24(3): I5561, 1982

7. Gomes, S. Incidência de reações sorológicas positivas para sífilis na população carcerária de Niterói. Arq. Bras. Med., 59(4): 275-8, 1985.

8. Guinsburg, R. et al. Sorologia positiva para síflis no período neonatal: prevalência em maternidade de nível secundá- rio; associação com fatores de risco maternos e com sorologia positiva para HIV-I. Rev. Assoc. Med. Bras., 39(2): 100-4, 1993.

9. Martino, H. et al. Sífilis congênita. Incidência, morbidade e mortalidade no Hospital Universitário Antonio Pedro (HUAP) J. Pediatr. (RJ), 52(3): I। I-2, 1982.

10. Monterrosa, E. Enfermedades de transmisión sexual en una regional del servicio de salud de Bogotá. Bol. Oficina Sanit. Panam., 99(3): 235-43, 1985.

I I. Parente, J.V. et al. Indicadores de risco gravídico no Piauí. Rev. Bras. Ginecol. Obstet; 6(5): 190-6, 1984.

12. Pires, I.C.P. \& Miranda, A.E.B. Prevalência e fatores correlatos de infecção pelo HIV e sífilis em prostitutas atendidas em Centro de Referência DST/AIDS. Rev. Bras. Ginecol. Obstet., 20(3): I5I-4, 1998.

13. Ramos, S.R.T.S. et al.Transmissão vertical das doenças sexualmente transmissíveis. Rev. Assoc. Méd. Bras., 3 / (9//0): I 88206, 1985

14. Reis, M.M. et al. Análise da sorologia para síflis utilizando o VDRL e a hemaglutinação passiva quantitativos e comparação dos resultados com o FTA-ABS. Rev. Bras. Patol. Clin., 22(6): 205-12, 1986

15. Souza, J.C.R.P. Comportamento sexual, DST/Aids e uso de drogas entre conscritos do exército brasileiro. Arq. Bras. Med., 68(2): 95-101, 1994. 